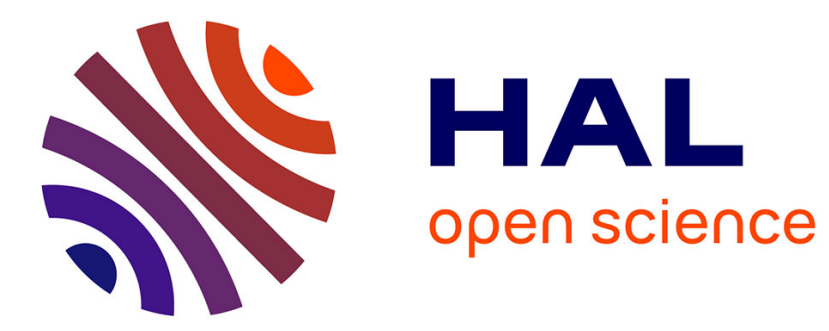

\title{
L'anthropologie au tribunal. Les revendications foncières des Aborigènes en Australie
}

\author{
Laurent Dousset, Katie Glaskin
}

\section{To cite this version:}

Laurent Dousset, Katie Glaskin. L'anthropologie au tribunal. Les revendications foncières des Aborigènes en Australie. Genèses. Sciences sociales et histoire, 2009, 74, pp.74-93. hal-00383037

\section{HAL Id: hal-00383037 \\ https://hal.science/hal-00383037}

Submitted on 30 May 2015

HAL is a multi-disciplinary open access archive for the deposit and dissemination of scientific research documents, whether they are published or not. The documents may come from teaching and research institutions in France or abroad, or from public or private research centers.
L'archive ouverte pluridisciplinaire HAL, est destinée au dépôt et à la diffusion de documents scientifiques de niveau recherche, publiés ou non, émanant des établissements d'enseignement et de recherche français ou étrangers, des laboratoires publics ou privés. 


\section{CA I R N}

chercher : repérer : avancer

Cet article est disponible en ligne à l'adresse :

http://www.cairn.info/article.php?ID_REVUE=GEN\&ID_NUMPUBLIE=GEN_074\&ID_ARTICLE=GEN_074_0074

L'anthropologie au tribunal. Les revendications foncières des Aborigènes en Australie

\section{par Laurent DOUSSET et Katie GLASKIN}

\section{Belin | Genèses}

2009/1 - $N^{\circ} 74$

ISSN 1155-3219 | ISBN 2-7011-5298-1 | pages 74 à 93

Pour citer cet article :

- Dousset L. et Glaskin K., L'anthropologie au tribunal. Les revendications foncières des Aborigènes en Australie, Genèses 2009/1, N 74, p. 74-93.

Distribution électronique Cairn pour Belin.

(c) Belin. Tous droits réservés pour tous pays.

La reproduction ou représentation de cet article, notamment par photocopie, n'est autorisée que dans les limites des conditions générales d'utilisation du site ou, le cas échéant, des conditions générales de la licence souscrite par votre établissement. Toute autre reproduction ou représentation, en tout ou partie, sous quelque forme et de quelque manière que ce soit, est interdite sauf accord préalable et écrit de l'éditeur, en dehors des cas prévus par la législation en vigueur en France. Il est précisé que son stockage dans une base de données est également interdit. 


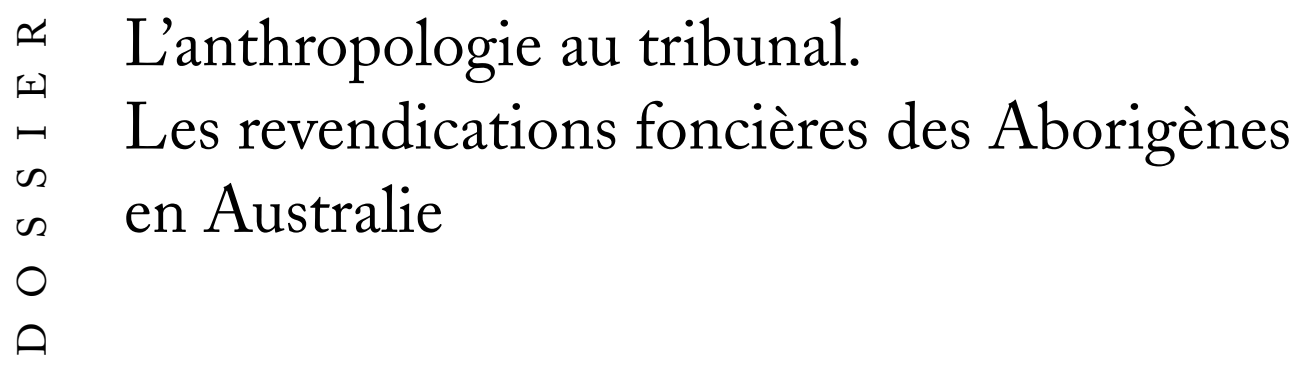

Laurent Dousset, Katie Glaskin

PP. 74-93

$\square$ ans l'anthropologie australianiste, comme au-delà, la nécessité de démontrer une "continuité culturelle» dans le cadre des revendications autochtones est confrontée à deux types de problèmes. Le premier est épistémologique. Il est le produit de l'autocritique et de l'évolution des savoirfaire de la discipline anthropologique elle-même. Il fait cohabiter des approches qui divergent et qui présentent l'objet d'étude sous des lumières variées. C'est de la confrontation entre les écoles de pensée, entre les théories de la forme et de la pratique, entre les fonctionnalismes et les structuralismes dont il s'agit. Ce premier type, aussi important soit-il pour l'évolution des sciences sociales, semble finalement mineur par rapport au second type de problèmes. Celui-ci relève de la morale, de l'éthique et du politique car c'est de cette ethnologie qui est nommée «appliquée» dont il est question. Cette anthropologie collecte et analyse des données dans une communauté, quelle qu'en soit la définition, pour ensuite les remettre sous forme de rapports et de témoignages à une institution tierce. Experte auprès des juges et des collectivités, conseillère auprès de sociétés minières ou d'organisations autochtones, elle porte sur son dos le lourd fardeau du destin d'une communauté tout entière. Sa responsabilité n'y est pas seulement scientifique, mais aussi sociale et politique. Des conclusions mal formulées ou même erronées n'engendrent pas seulement des discussions théoriques limitées au monde de la recherche, mais risquent de susciter des drames qui dépassent le champ d'action de la discipline. Dans le cadre de ce second type de problèmes, le travail ethnologique est noyé dans une dimension tout autre que celui qui est soumis à la seule critique des confrères.

Une distinction catégorique de ces deux types de problèmes est pourtant discutable, tout particulièrement dans le contexte australien. La discipline y est fortement impliquée dans les processus juridiques et politiques contemporains et les épistémologies anthropologiques sont inséparables de leurs mises en pratique. 
Certains modèles et concepts théoriques produits jadis par l'anthropologie académique furent même à l'origine des cadres juridiques qui définissent les droits des populations indigènes, notamment dans le contexte des revendications foncières. Les sciences sociales appliquées contemporaines doivent désormais savoir s'exprimer en des termes définis par ces modèles dits classiques. De plus, les institutions auxquelles le chercheur apporte son savoir s'emparent des théories anthropologiques, les interprètent comme des vérités incontournables et affaiblissent l'expertise contemporaine lorsqu'elle propose des modèles alternatifs. La pratique anthropologique est ainsi située au cœur d'un affrontement lourd de conséquences pour les communautés. Un affrontement qui oppose une discipline dont les grilles d'analyses évoluent comme dans toute science, et une corporation d'acteurs antagonistes - juristes en particulier - qui, instruits par ces mêmes savoirs mais sans les faire évoluer, s'oppose à toute proposition qui divergerait des modèles classiques. "Anthropologie» et «Droit», à défaut d'être presque devenus complices, sont aujourd'hui démarqués et souvent même s'affrontent autour de questions qui constituent le noyau même de la discipline ethnologique.

C'est ce contexte particulier que nous nous proposons d'exposer en discutant un exemple singulier et révélateur à la fois: une demande de compensation de la part de membres du groupe aborigène des Pitjantjatjara du Désert de l'Ouest à l'encontre de l'État (le Territoire du Nord). Au cours de cette procédure juridique (Jango vs Northern Territory of Australia), connue depuis sous le nom de Yulara case, le juge décida en 2006 que la demande de compensation était infondée, donnant raison au Territoire du Nord. Comme nous le verrons, les arguments que le juge fit siens pour rejeter la demande sont particulièrement dérangeants. Ils montrent qu'il s'est emparé des modèles anthropologiques classiques pour anéantir toute preuve contemporaine apportée devant la Cour. Après un exposé des principes qui règlent les revendications, foncières en l'occurrence, et un bref rappel de l'historique de la charpente permettant aux Aborigènes de faire valoir leurs revendications, nous montrerons que le juge dans le cas Yulara se constitua lui-même en expert, décrivant une société à laquelle les Pitjantjatjara ne ressemblent pourtant pas. Soulignant une disparité entre la société décrite par les anthropologues contemporains et le modèle qu'il s'était reconstruit par ses lectures, le juge affirma que les Pitjantjatjara constituent un groupe dont les fondements socioculturels s'opposeraient à sa propre tradition, annihilant de fait tout droit à la compensation.

\section{Le processus juridique des revendications foncières}

Nous reviendrons sur l'origine des cadres qui permettent aux Aborigènes de soumettre des revendications foncières. Il est utile d'en exposer d'abord les principes de fonctionnement. La lecture de la critique qui suivra en sera d'autant plus 
éclairée. Un constat simple, mais fondamental à ce sujet, est que le système juridique australien se fonde sur le principe de la jurisprudence. Les textes législatifs certes existent, mais ils ne font que décrire les cadres généraux des principes de droit et de procédure. Chaque procès et chaque verdict affinent, nuancent ou même modifient parfois considérablement l'applicabilité de ces textes. Ce sont les processus juridiques qui pèsent le plus car ils deviennent des références pour les procès ultérieurs. Tout nouveau procès prend en compte les conclusions de procès similaires qui l'ont précédé, mais fait aussi appel à des témoins experts qui exposent les particularités du procès afin de faire évoluer la jurisprudence.

Dans le cas des revendications foncières, les parties qui s'opposent sont l'État et des organisations représentant les Aborigènes. Ces organisations, nommées Representative Bodies, sont des organismes régionaux reconnus par l'État fédéral comme étant des interlocuteurs privilégiés. Sous le contrôle de leurs avocats, ils embauchent des chercheurs en sciences sociales, en grande majorité des anthropologues, qui deviennent ainsi leurs experts. Leur participation dans le processus juridique se joue à deux niveaux. Dans un premier temps, ces chercheurs - certains travaillant à leur propre compte, les autres étant des chercheurs académiques - préparent les informations nécessaires à la négociation ou à la prise de décision par la Cour qui jugera ainsi de la recevabilité de la demande. Dans cette première phase, ils rédigent un Connection Report. L'objectif de ce texte est de démontrer la nature et la continuité des pratiques culturelles traditionnelles. En effet, les revendications ne sont admissibles que si les pratiques contemporaines reflètent des pratiques dites "traditionnelles", c'est-à-dire si la preuve est faite que l'organisation sociale et territoriale contemporaine ne diverge pas de manière structurelle de celles à l'œuvre avant le contact avec les Britanniques. Cette exigence fait partie du cadre législatif général organisant les revendications aborigènes. Le rapport doit ainsi, en dressant des généalogies nominatives et extensives, démontrer la continuité et la cohérence physique du groupe demandeur. Il doit également démontrer une continuité culturelle en exposant la nature des liens qui associent ces généalogies au foncier: mythologiques, rituelles, économiques, etc. En résumé, il s'agit de montrer par le recueil de données sur le terrain, par l'analyse d'archives lorsqu'elles existent et par la juxtaposition de ces données avec la littérature anthropologique, que la relation du groupe demandeur à l'étendue foncière revendiquée n'a ni été interrompue, ni substantiellement modifiée, depuis la colonisation. Le Connection Report, véritable monographie ethnologique, est ensuite soumis à la partie adverse, c'est-à-dire à l'État.

L'État à son tour recrute des anthropologues. Inversement, leur tâche est de démontrer que la continuité physique et culturelle du groupe en question ne peut être affirmée. S'ils considèrent que la démonstration faite dans le Connection Report est recevable, et que l'État décide de suivre leurs conseils, alors l'affaire est différée pour négociation devant un tribunal particulier, le National Native Title Tribunal (NNTT). Ce tribunal ne rend aucun jugement. Il encadre et accom- 
pagne les négociations entre le Representative Body et l'État. Si un accord est trouvé, alors il est question d'une restitution allouée par consentement. Si, au contraire, les anthropologues recrutés par l'État rejettent de manière substantielle les démonstrations fournies par les anthropologues du Representative Body, alors le cas est différé devant la Cour fédérale.

Seuls les anthropologues recrutés par les Representative Bodies effectuent en concertation avec leurs "clients" des enquêtes sur le terrain. Les autres travaillent sur archives, citent la littérature existante et analysent le Connection Report sur sa cohérence interne. Il va sans dire que ceux qui travaillent pour l'État sont souvent les mêmes d'un procès à l'autre et qu'ils émettent des opinions sur de multiples régions et sociétés aborigènes, sans toujours bien connaître les particularités locales. En effet, ceux qui acceptent d'offrir leurs services à l'État sont plus rares que ceux qui décident de travailler pour les Representative Bodies. Les seconds incluent le plus souvent des spécialistes de l'aire culturelle en question et bénéficient déjà d'expériences de terrain.

Dans une seconde étape et si la restitution par consentement ne peut avoir lieu, les rapports anthropologiques sont déposés auprès de la Cour fédérale. D'autres acteurs peuvent alors rejoindre le procès et se constituer en parties adverses: des sociétés minières, par exemple. Elles recrutent également des anthropologues qui travaillent eux aussi sur littérature et archives, sans effectuer d'enquêtes de terrain. Les divers anthropologues seront convoqués par le juge comme experts au cours du procès où ils apparaitront devant la barre. Le concept d'expert implique ici un degré d'objectivité et de neutralité explicité dans les textes qui règlent les procédures de la Cour fédérale (Glaskin 2004; Morphy 2006):

1. Un expert témoin à la Cour a la tâche d'assister la Cour dans le domaine qui concerne son expertise.

2. Un expert témoin à la Cour n'est pas l'avocat d'une des parties impliquées.

3. Le devoir premier d'un expert témoin à la Cour est envers la Cour, et non envers la personne engageant l'expert.

En d'autres termes, si dans le Connection Report les anthropologues se soumettent de fait à la stratégie développée par les avocats du Representative Body, réunissant les informations et proposant des conclusions qui, selon eux, aideront à soutenir leur cause, dans le cadre du procès, par contre, ces mêmes anthropologues sont soumis au principe d'objectivité scientifique. À côté des Aborigènes eux-mêmes, les anthropologues constituent les personnes clefs appelées à la barre. Ajoutons encore, avant de clore ce très rapide survol des mécanismes juridiques, que les juges qui se chargent de ces procès ne sont pas des spécialistes euxmêmes. Ils n'ont souvent pas l'habitude de ce genre de situations et apprennent (ou n'apprennent pas) au cours du procès à manier les concepts de la discipline.

Résumons les points essentiels. Le système juridique se fonde sur la jurisprudence, ce qui dans le cas de litiges différés devant la Cour fédérale confère aux 
juges un pouvoir considérable dans la modélisation des jugements ultérieurs. Les données et la théorie anthropologique constituent les sources d'informations principales. Au travers de ces sources, les juges décident s'il y a continuité culturelle et physique. Les informations qui permettent au juge de prendre ces décisions sont fournies par les anthropologues avant et pendant le procès. Les juges formulent leurs conclusions en des termes quasi anthropologiques qui deviennent jurisprudence pour les cas ultérieurs. Nous voyons donc, dès la toute première revendication foncière, que les conclusions des juges ont déterminé les cadres des jugements ultérieurs. Nous constatons aussi que l'anthropologue, lorsqu'il est appelé devant la Cour, est donné comme un scientifique objectif et non comme un témoin au service d'une des parties. Nous verrons maintenant que ces deux constats trouvent leurs origines dans les mêmes évènements historiques et puisent leurs sources dans la même théorie anthropologique; et nous observerons à quel point cette intégration précoce entre anthropologie et jurisprudence rend aujourd'hui difficile la modification des principes de bases qui furent définis jadis.

\section{De la fabrication de l'anthropologie appliquée «naturaliste»}

Quelques remarques sur l'émergence de l'anthropologie appliquée, puis sur l'intégration des modèles anthropologiques dans la jurisprudence, permettront de mieux saisir les sources auxquelles le juge de Yulara fit référence. Si l'implication de l'ethnologie dans la gestion des colonies fut discutée dès la création de la Aborigines Protection Society en 1838 (Reining 1962), ce n'est qu'au début du XXe siècle que deux types d'approches se dessinent nettement. La première, que nous appellerons «naturaliste», défendue par Alfred R. Radcliffe-Brown, définissait l'anthropologue comme un scientifique objectif capable de produire des modèles durables et extrapolables. L'expert ne devait occuper qu'une fonction scientifique et ne pas s'inquiéter des conséquences sociales que son expertise pouvait provoquer (Keen 1999): il est au service du colonialisme (Radcliffe-Brown 1929; McCall 1980).

L'autre approche, défendue par Bronislaw Malinowski (1929, 1938), Edward E. Evans-Pritchard (1950) ou encore Alfred L. Kroeber (Keen 1999: 47) ne croyait pas, au contraire, à la capacité d'une objectivité parfaite et concevait l'anthropologie comme intimement imbriquée dans des processus politiques. L'anthropologue pouvait certes produire du savoir et tirer des conclusions scientifiques, mais il devait aussi mettre en valeur son action politique. Enrichi par son expérience de terrain, il se trouverait dans une position idéale pour intervenir dans la nature des transformations sociales induites par l'occidentalisation des pratiques.

Étant donné la position dominante que Radcliffe-Brown occupait dans l'académie, ce fut sa position qui vint à définir la marche à suivre en Australie. L'approche «naturaliste» devint la norme pour toute «bonne» participation 
anthropologique à la vie sociopolitique, s'opposant à la «mauvaise» pratique qui consistait à prendre position dans les conflits et à exposer une opinion morale et idéologique. Il s'agissait de pratiquer une anthropologie appliquée qui devait être capable de fabriquer du savoir permanent tout en faisant abstraction du contexte de sa propre production. Nous avons vu que c'est cette définition qui fut reprise dans les guides de la Cour fédérale pour définir le rôle de l'anthropologue, et nous verrons que c'est aussi de cette permanence des modèles produits par l'anthropologie «naturaliste» que le juge de Yulara se servira pour se constituer en tant qu'expert dans un premier temps, et pour privilégier les sources anciennes à l'encontre de l'expertise contemporaine dans un second temps.

L'approche «naturaliste» eut néanmoins des retombées bénéfiques. Dans un contexte politique particulièrement négligeant à l'égard des peuples indigènes, elle réussit à opposer une "vérité» à une autre et à mettre sur un pied d'égalité les modèles juridico-politiques dominants et les modèles sociaux autochtones. Déployer l'approche "naturaliste» permettait de décrire des règles et des structures sociales comme s'il s'agissait de lois, légitimant ainsi la participation de l'anthropologie dans la définition des cadres juridiques. Voyons quels furent les modèles qui furent intégrés dans ces cadres.

\section{Le contexte des revendications foncières}

C'est le principe de la descendance unilinéaire, pièce maîtresse du structurofonctionnalisme de Radcliffe-Brown, qui devint le modèle général intégré dans les jurisprudences des revendications foncières (Gumbert 1984). Selon ce postulat, l'anthropologue, et par voie de conséquence la Cour, sont à la recherche de structures politiques qui maintiennent des équilibres territoriaux. Ces équilibres s'articulent autour de principes de descendance qui organisent la société en groupes locaux et en clans. La tâche de l'expert fut ainsi de définir ce qui constitue localement «la horde» selon Radcliffe-Brown (1956) ou «le groupe de descendance local» selon Leach (1951). Pour l'exprimer dans les termes de la Cour, lorsque l'anthropologue recruté par le Representative Body réussit à démontrer que des clans patrilinéaires et propriétaires fonciers étaient à la base de l'organisation traditionnelle d'un groupe, et qu'il apporte également la preuve que cette organisation clanique est toujours à l'œuvre aujourd'hui, la continuité culturelle et physique se voit, à quelques détails mineurs près, prouvée d'emblée et la revendication donc recevable.

L'intégration de ces principes dans la jurisprudence trouve ses origines dans la toute première revendication foncière. En 1968, les Yolngu de la terre d'Arnhem, propriétaires traditionnels de la Gove Peninsula où s'était installée la compagnie minière Nabalco, engagèrent des poursuites à l'encontre de cette société afin de récupérer leurs droits fonciers. Cette plainte (Milirrpum vs Nabalco Pty 
Ltd 1971), connue sous le nom de Gove land rights case, fut entendue devant la Cour fédérale en 1970. La revendication des Yolngu ne fut pas couronnée de succès. Néanmoins et pour la première fois, le juge admit l'existence d'un système de droit coutumier. Cette conception radicalement nouvelle selon laquelle la société aborigène a la capacité d'être organisée par des systèmes de droit donna lieu à une commission qui diligenta des enquêtes. Le processus déboucha sur l'Aboriginal Land Rights (Northern Territory) Act 1976 (ALRA) qui décrit le cadre permettant la récupération foncière de territoires inoccupés. Les anthropologues experts dans le cas de Gove - des personnalités célèbres dans la discipline comme Ronald M. Berndt et William E. Stanner - développèrent l'articulation d'un système coutumier autour de clans localisés, propriétaires et exploitants du foncier à la fois: un système unilinéaire qui fit son chemin dans l'ALRA. Cette articulation fut pourtant contredite par les témoignages des Aborigènes euxmêmes pendant le procès (Gumbert 1984: 78-81). Mais la vision radcliffebrownienne selon laquelle la succession généalogique serait le principe organisateur des droits fonciers fut néanmoins reprise dans le Land Rights Act; par exemple dans la section 3(1) où les "propriétaires coutumiers» sont définis comme un "groupe de descendance local». Les Aborigènes qui entreprenaient dès lors des démarches devaient prouver qu'ils étaient organisés selon ces principes. Comme l'expliquent James F. Weiner et Katie Glaskin:

«En raison de la manière dont elle permet d'assigner des personnes à des groupes sociaux définis sans aucune ambiguïté théorique, la descendance unilinéaire comme principe de recrutement social est devenue "l'institution indigène préférée" de l'appareil législatif [...] Il est inutile de souligner que le principe de descendance unilinéaire se soumet plus facilement aux conditions de légitimation exigées par l'État que d'autres mécanismes de recrutement social et d'affiliation territoriale $[\ldots] »(2007: 9-10)$.

L'ALRA n'était applicable que dans le Territoire du Nord. L'étape suivante fut le Native Title Act 1993 (NTA), conséquence de la décision de la Haute Cour dans le cas Mabo contre l'État du Queensland (Mabo E Ors vs Queensland, n ${ }^{\circ}$ 2, 1992). Suite à ce cas, l'existence d'un «titre foncier coutumier» fut pour la première fois inscrite dans la législation.

\section{De la tradition et de la continuité}

Par le biais du concept de Native Title («titre coutumier»), le NTA définit le contexte dans lequel la reconnaissance juridique de droits aborigènes tels qu'ils seraient définis par leurs propres lois coutumières peuvent être pris en compte. Ces lois coutumières constituent la base sur laquelle le titre peut être légalement reconnu. Confiné largement à ce qui est appelé Vacant Crownland, c'est-à-dire 
des surfaces qui font partie du domaine public, le NTA décrit aussi les obligations auxquelles les demandeurs sont invités à se soumettre pour envisager la restitution. Les groupes qui s'engagent dans cette procédure doivent satisfaire plusieurs conditions. D'abord celle d'être les descendants directs du groupe qui résidait et possédait selon la loi coutumière le territoire revendiqué au moment de l'acquisition de la souveraineté britannique. Le modèle dominant qui permet de satisfaire cette exigence est le principe de la descendance unilinéaire décrit plus haut. Ensuite ils doivent être capables de démontrer que ces lois et coutumes sont toujours celles qui organisent la collectivité contemporaine. Il leur est ainsi nécessaire de faire la preuve d'une continuité culturelle et généalogique ininterrompue depuis la colonisation. Enfin, dernière mais importante condition, les demandeurs doivent démontrer qu'ils constituent une société telle qu'elle fut définie dans la jurisprudence du cas Yorta Yorta.

Le cas qui entraîna les modifications les plus significatives et contraignantes du NTA, diminuant considérablement l'envergure du concept même de «titre coutumier", fut celui de Western Australia vs Ward (en 2000 et 2002), nommé le «cas Ward». Ce cas concernait les Miriuwung et les Gajerrong dans les Kimberleys du nord-ouest australien et instaura la notion de bundle of rights, littéralement un ensemble ou paquet de droits (Glaskin 2003). Dans le cadre de la législature originale, un groupe aborigène devait démontrer être l'héritier d'un système global de lois coutumières. Il s'agissait d'illustrer une continuité culturelle dans laquelle les transformations sociales induites par la colonisation ne seraient que superficielles et non structurelles. Le cas Ward, par contre, stipule que le droit foncier est constitué d'un ensemble de droits qui doivent être démontrés individuellement. Prouver que la société dite traditionnelle pratiquait la chasse au moment de la souveraineté britannique, par exemple, peut conduire au droit à la chasse aujourd'hui. Les anthropologues qui rédigent le Connection Report doivent maintenant démontrer la continuité de tout un panel de pratiques ancestrales afin de pouvoir acquérir les droits un par un, au lieu de n'avoir à démontrer que l'existence d'un droit coutumier global comme cela était le cas auparavant. Ce contexte général étant posé, revenons maintenant au cas qui nous intéresse plus particulièrement ici, celui de Yulara.

\section{Le cas Yulara: quand les modèles anthropologiques se retournent contre les anthropologues}

Lorsque les anthropologues de l'État considèrent que le Connection Report ne prouve pas la continuité culturelle et physique, les litiges sont jugés devant la Cour fédérale. En appel, ils sont jugés devant la Cour fédérale plénière (trois juges) et en dernière instance devant la Haute Cour. Dans chacun de ces cas, la référence au modèle unilinéaire est sous-jacente aux rapports fournis par les 
experts, car c'est celui qui répond le mieux à la jurisprudence des revendications précédentes. Lorsque ce modèle est difficilement applicable sur le terrain, les chercheurs sont néanmoins amenés, sous la pression des avocats, à présenter leur matériel en favorisant l'existence de principes généalogiques qui permettraient de définir un ensemble de règles formelles susceptibles d'être comprises et acceptées par la Cour. Tel ne fut pas le cas lors de la demande des Pitjantjatjara, où les anthropologues experts du Representative Body prirent le risque de présenter le système tel qu'il existe effectivement sur le terrain. Nous le verrons plus loin, ce système n'a aucune ressemblance avec le modèle unilinéaire.

Yulara fut le premier cas de compensation dans le cadre du Native Title. Il ne s'agissait pas d'une demande de restitution foncière, mais d'une requête de dédommagement fondée sur l'affirmation que le village touristique de Yulara y avait été établi sans considération des droits indigènes. Qu'il s'agisse d'une demande de compensation et non de restitution foncière ne change en rien notre propos, puisque dans les deux cas les procédures juridiques et les modalités de la preuve sont identiques. La demande de compensation fut d'abord rejetée devant la Cour fédérale en première audience. Un jugement en appel, lui aussi sans succès, fut entendu en novembre 2006 devant la Cour fédérale plénière cette fois. Une ultime décision fut rendue le 6 juillet 2007 en appel.

Yulara provoqua des débats et polémiques anthropologiques (Dousset et Glaskin 2007) qui s'articulaient autour de deux questions centrales. La première concerne la notion de «société» telle qu'elle est définie et exigée par le NTA et la jurisprudence de Yorta Yorta. Peut-on l'appliquer au bloc culturel du Désert de l'Ouest dont les Pitjantjatjara font partie et où pourtant les premières ethnographies avaient déjà souligné la grande fluidité sociale et territoriale et où l'absence de corporations comme le "clan", ou même la "tribu», fut mise en avant? La seconde question interroge l'opportunité des modèles classiques dits radcliffebrowniens dans une structure sociale qui ne semble pas s'y prêter. La question de l'applicabilité de ces modèles se métamorphosa, devant la Cour et dans les débats anthropologiques qui suivirent, en un conflit entre les avocats des preuves jugées valides par leur ancienneté et les partisans des preuves nouvelles apportées par des travaux récents (Sutton 2006, 2007; Sansom 2007; Burke 2005, 2007; Glaskin 2007; Keen 2007; Morton 2007; Sackett 2007; Dousset et Glaskin 2007). Notons que parmi ces chercheurs, Peter Sutton était l'un des anthropologues recrutés par l'organisation aborigène, Basil Sansom l'expert recruté par le Territoire du Nord et John Morton celui représentant l'État fédéral. Mais notons également que l'État fédéral a choisi, en dernière instance, de ne pas opposer d'expertise anthropologique à celle fournie par les autres anthropologues.

Après avoir analysé les rapports et entendu les témoignages, le juge traita les deux questions de manière conjointe. D'abord il accepta l'existence de la notion de société pour le Désert de l'Ouest car il considérait qu'il n'était pas nécessaire de définir des division internes pour constater que ses habitants étaient régis par un 
ensemble de règles et de lois qui leur est propre et qui organise la vie collective. À l'encontre de l'avis des experts qui avaient effectué des terrains récents chez les Pitjantjatjara, le juge décida toutefois que ces règles et lois coutumières qui constituent la société étaient fondées sur la patrilinéarité, c'est-à-dire un système unilinéaire. Or, ajouta-t-il, cette patrilinéarité a disparu aujourd'hui. En conséquence, il conclut sur une discontinuité culturelle entre la société dite traditionnelle et la société contemporaine. Selon les termes du NTA, cette discontinuité évacue toute opportunité de compensation et de revendication.

«Les preuves n'ont pas été faites que les lois et coutumes observées et reconnues aujourd'hui sont suffisamment proches de celles observées et reconnues par les habitants du Désert de l'Ouest au moment de la souveraineté. En particulier, je conclus que les lois et coutumes traditionnelles du Désert de l'Ouest suivaient un principe de descendance patrilinéaire, qui est toutefois largement absent des pratiques décrites dans les témoignages»

(Jango ws Northern Territory of Australia [2006] FCA 318, \$11).

Le juge renforça son argumentation en soulignant une désorganisation des témoignages, critère qui lui permirent d'affirmer une forte déculturation et donc une absence de droits coutumiers. L'absence d'homogénéité, c'est-à-dire l'absence d'un modèle unilinéaire précis et clair selon le schéma radcliffe-brownien, était devenue la preuve d'une déculturation.

«Si les preuves indigènes avaient de manière homogène énoncé un ensemble de lois et de coutumes particulières, on aurait pu déduire que ces lois et coutumes décrites par les témoins sont restées identiques en substance depuis la souveraineté [...] En effet, le fait que, en temps modernes, les personnes ont apparemment adhéré à des versions de lois et coutumes si différentes suggère que les changements qui ont eu lieu depuis la souveraineté ne sont pas de simples "adaptations"»

(Jango vs Northern Territory, \$272).

La lecture des témoignages aborigènes et des rapports d'experts montre que ce qui fut exposé ne reflétait certes pas un modèle unilinéaire fondé sur des clans et des droits de propriété inaliénables, mais que cette hétérogénéité correspondait par contre tout à fait à la réalité ethnographique du Désert de l'Ouest.

\section{De la suprématie des modèles mythiques sur la réalité ethnographique}

De nombreux chercheurs ont critiqué la notion de "propriétaire traditionnelle» telle qu'elle avait été définie dans le ALRA d'abord, puis reprise dans le NTA, car elle impliquait des contraintes importantes sur la conduite des enquêtes de terrain et sur les formulations des demandes de restitution foncière 
(Maddock 1982; Rumsey 1996). Comme nous l'avons évoqué, ce modèle anthropologique trouve ses origines dans le structuro-fonctionnalisme qui était dominant dans l'analyse de la parenté et de l'organisation territoriale à l'époque où les procédures et textes législatifs furent établis (Weiner et Glaskin 2006: 2). Le primat des groupes de descendance suscita des difficultés à cause du paysage varié des organisations socioterritoriales australiennes. Certes, Lester R. Hiatt s'était empressé dès 1962 de démontrer que le modèle était inapplicable (1962) et l'on se souviendra de sa contribution à la première conférence «Man the Hunter» (Hiatt 1968) où, face à Radcliffe-Brown et Lévi-Strauss, il déconstruisait les théories dominantes pour les remplacer par une ethnographie reflétant les dynamiques sociales. Mais on se souviendra aussi de la réponse de Claude LéviStrauss qui ne trouva comme argument central que celui, classique sous sa plume, de la déchéance culturelle et de la primauté de la règle sur la pratique.

«Si les observations récentes de Hiatt de ce qui reste d'une tribu australienne qui s'effondre portent davantage de poids que toute la littérature plus ancienne, alors brûlons les livres. [...] Je conviens qu'il est important de découvrir ce qui se produit effectivement dans les sociétés, malgré les règles proclamées. Cependant, mon travail sur la parenté pour la plupart des cas était concerné par un problème différent: celui de découvrir la signification des règles, qu'elles soient appliquées ou non»

(Lévi-Srauss 1968: 211).

Ce qui est significatif dans cette citation est le renvoi qu'opère Lévi-Strauss vers des règles et des modèles dont la connexion avec la réalité ethnographique lui semble secondaire. Pour les juges, pourtant, les modèles reflètent effectivement les règles qui organisent la société et ne sont pas seulement des abstractions théoriques. Il faut également souligner dans la citation de Lévi-Strauss la primauté des sources anciennes - qu'elles ne soient constituées que de quelques lignes de missionnaires comme cela est souvent le cas ou qu'elles soient plus riches - sur les terrains plus récents mais de longue durée. Le critère de l'ancienneté de la source est ainsi placé en tête de la hiérarchie des preuves anthropologiques, immédiatement suivi de la modélisation théorique. Les ethnographies descriptives et les terrains récents et intensifs sont relégués en dernière position car associés à une situation d'effondrement culturel.

Nous l'avons évoqué en introduction: cette hiérarchie et les discussions théoriques qui en découlent relèvent de débats internes à l'anthropologie et n'œuvrent généralement pas à l'extérieur du domaine académique. Le fait que Lévi-Strauss et Hiatt se soient affrontés dans une arène académique ne devrait que peu intéresser les Aborigènes eux-mêmes. Tel n'est toutefois plus le cas en Australie où juges et avocats connaissent et lisent les anthropologues et où ils interprètent l'ancienneté et le modèle comme des vérités observées qui doivent perdurer pour pouvoir faire la preuve de la continuité culturelle. Les controverses anthropologiques se sont échappées du domaine académique pour s'installer durablement dans la vie 
publique. C'est donc en se fondant sur l'universalité du modèle classique défendu par Radcliffe-Brown que le juge de Yulara proclama l'existence de groupes de descendance patrilinéaires dans le Désert de l'Ouest. Montrer que la décision du juge est erronée nécessite maintenant l'exposé de la réalité ethnographique.

Les Pitjantjatjara sont l'un des quelque quarante groupes dialectaux composant ce qui depuis l'anthropologue Berndt (1959) est appelé le bloc culturel du Désert de l'Ouest couvrant plus de 600000 kilomètres carrés. Depuis qu'elles sont étudiées, ces sociétés ont posé des problèmes importants à l'anthropologie. Si ailleurs les modèles radcliffe-browniens, la conception «africaniste» de la notion de tribu, ou encore les idées structuralistes de la parenté pouvaient être appliqués (non sans parfois procéder à des distorsions importantes de l'ethnographie), le Désert de l'Ouest, par contre, semblait échapper à toute unification théorique de l'ethnographie australienne. Ces sociétés furent ainsi largement définies en termes «d'absence» (Dousset 2003). Absence de catégories sociales qui ailleurs semblaient organiser la parenté et la vie sociale; absence de clans; absence de groupes locaux; absence de principes de descendance; absence d'unités globales comme la «tribu», etc. Lévi-Strauss (1967: 231 et suiv.), visiblement perplexe devant ce système, l'avait classé parmi les systèmes «aberrants ».

Ces problèmes théoriques sont la conséquence de l'utilisation de sources ethnographiques peu fiables pour cette région, à commencer par les travaux d'Adolphus P. Elkin (1931, 1938-1940). C'est à la demande de Radcliffe-Brown, alors directeur du seul département d'anthropologie en Australie à Sydney de 1925 à 1931, qu'Elkin partit en 1930 pour effectuer un inventaire rapide des systèmes de parenté à l'ouest et au nord de la rivière Murray en Australie du Sud. Pendant cette année, il visita un grand nombre de tribus et d'aires culturelles et récolta une quantité importante de données certes intéressantes mais peu approfondies. Linfluence de Radcliffe-Brown sur ses collègues était considérable, comme en témoignent ses échanges avec un autre anthropologue, Donald Thomson (Peterson 2006). Elkin n'allait pas faire exception à ce sujet et il s'efforça de décrire les multiples systèmes de parenté qu'il rencontra lors de son séjour en appliquant la grille typologique de son maître. Pour le Désert de l'Ouest, toutefois, ses tentatives laissent entrevoir de nombreuses difficultés d'interprétation (Dousset 2002, 2003). Ne réussissant pas à distinguer et à nommer clairement des groupes spatiaux et à comprendre l'organisation socioterritoriale (Elkin 1938-1940: 203), il nomma l'ensemble situé à l'ouest de la tribu des Aranda les "Aluridja» (ibid.: 424, 204) ou plus simplement «le groupe de l'ouest» (1931: 60) afin d'en souligner une apparente similarité culturelle sans en définir son contenu. Enfin, il tenta d'expliquer la fluidité territoriale et l'absence de véritables démarcations tribales (1938-1940: 203, 295 et suiv.) comme étant une conséquence des migrations. Certes, les migrations et surtout les échanges et diffusions de traits culturels sont et ont été importants dans cette région (Dousset 2005). Elles ne permettent toutefois pas d'expliquer convenablement l'organisation particulière qui y règne. 
Prenons l'exemple du groupe dialectal des Ngaatjatjarra, voisins des Pitjantjatjara, que nous connaissons pour y avoir effectué des terrains de longue durée. Un groupe dialectal n'est pas une tribu, ni même un ensemble de personnes qui peuvent être définies par leur appartenance linguistique. Les linguistes ont montré que tracer des frontières géographiques autour des dialectes n'est pas pertinent pour deux raisons essentielles. La première est la grande mobilité des individus et familles, la seconde est due aux différences linguistiques graduelles au détriment d'une différenciation claire et articulée. Il est impossible de s'accorder même sur le dialecte véhiculé par telle ou telle personne (Miller 1971; Douglas 1971). Berndt déjà proposait - en réponse à Elkin, Tindale et autres prédécesseurs qui tentaient de reconstruire dans le Désert de l'Ouest un modèle «australien» classique - de nommer les unités même temporaires que l'on pouvait y observer «sociétés» plutôt que «tribus».

Les appellations de ces groupes dialectaux sont ambiguës, car elles dénotent des idiosyncrasies relatives plutôt que des ensembles clos. Pitjantjatjara, par exemple, signifie ceux qui disent pitja pour signifier le verbe «aller». Ils se distinguent des Yankunytjatjara qui utilisent yankuny pour le même verbe. Les Ngaatjatjarra, toutefois et même s'ils utilisent pitja pour dire «aller», utilisent ngaatja pour le mot «ceci», se distinguant ainsi des gens à l'ouest qui utilisent ngaanya pour désigner la même chose. Au sein même des Pitjantjatjara, notamment ceux de l'ouest, les Ngaatjatjarra distinguent les Nyngatjatjara, car ces derniers disent nyangatja pour «ceci»; mais tous disent pitja pour «aller», etc. Ce petit jeu de critères de distinction qui inclut tantôt les uns, tantôt les autres, peut être mené pour l'ensemble du Désert de l'Ouest. Ces appellations furent néanmoins fixées suite à la présence des missionnaires dès les années 1920 dans certaines régions, et surtout avec l'arrivée de l'administration et de ses recensements où renseigner la case «appartenance tribale» était une obligation. Pour les Ngaatjatjarra, avant leur premier contact durable avec l'Occident en 1956, les noms collectifs suivaient plutôt le principe de la toponymie. Patjanguraru, par exemple, désignait l'homme qui vivait sur le site de Patjara (nguraru étant un locatif) avec ses trois femmes et sa filiation, constituant ainsi le groupe de résidence de Patjara.

Nous parlons donc ici de groupe de résidence et de groupe régional, car ce qui est plus généralement connu sous l'expression «groupe local» ou «clan localisé», faisant références aux principes unilinéaires, ne trouve pas de correspondant dans le Désert de l'Ouest. Les individus vivaient jusque dans les années 1960 en petits groupes qui comprenaient la famille nucléaire avec un ou plusieurs enfants adoptés et parfois des vieillards. Il s'agissait de groupes domestiques qui consommaient en commun ce qu'ils produisaient. Plusieurs de ces groupes de résidence constituaient ce que nous appelons des groupes régionaux. Il s'agissait d'un ensemble de personnes qui, par leur proximité géographique et souvent généalogique, se rencontraient régulièrement pour échanger des informations et des biens ainsi que pour pratiquer en commun des rites associés aux espèces et aux sites sur lesquels ils vivaient. 
Ces ensembles de groupes de résidence, que nous appelons groupes régionaux, étaient en principe exogames. Si l'exogamie contenait à la fois le critère spatial et généalogique, la règle formulée reflétait surtout la spatialité, car l'idéal était d'établir des alliances maritales avec des familles distantes, résidant avec d'autres groupes régionaux. Toutefois, même si ces groupes étaient composés d'unités résidentielles et même si la préférence maritale était l'exogamie forte, les membres de ces groupes n'étaient pas néanmoins et automatiquement les propriétaires des étendues sur lesquelles ils nomadisaient. L'affiliation territoriale s'acquérait et s'acquiert encore aujourd'hui par l'accumulation de critères et non par leur héritage. Elle est d'ailleurs toujours exprimée en faisant référence à des sites particuliers - dont certains peuvent être loin du lieu de résidence - et non à des surfaces ou des frontières. Le nombre de sites auxquels une personne est associée croît avec le parcours individuel. Le lieu de conception (c'est-à-dire l'endroit où l'on pense que l'enfant-esprit a pénétré la mère pour s'y loger et devenir un être humain), le lieu de naissance, le lieu de résidence prolongée, les ressemblances corporelles avec des héros mythiques, les savoirs acquis sur des mythes et des chants qui concernent des sites particuliers, le lieu d'initiation, les lieux de résidence prolongée des parents et grands-parents... sont des critères qui participent à la capitalisation d'un droit à la parole sur un ou plusieurs sites. L'espace est ainsi progressivement humanisé et la géographie socialisée. Les personnes obtiennent l'un de leurs noms personnels du site auquel ils s'associent et ce site peut être désigné en citant la personne. Dans d'autres contextes et pour d'autres locuteurs, toutefois, ce même site peut être désigné par le nom d'une autre personne encore. Aucun individu ou groupe d'individu n'est le propriétaire exclusif d'un site et aucun site ne reflète l'appartenance d'un seul individu. La propriété collective ou clanique, héritée et transmissible, la propriété exclusive et le pouvoir d'interdire l'accès à des lieux ou régions tout entières, critères propres aux groupes de descendance locaux radcliffe-browniens, ne correspondent à rien dans cette région.

Certains auteurs ont associé ce système foncier fluide aux conditions écologiques extrêmes qui règnent dans la région. En effet, un système territorial fondé sur des clans patrilinéaires qui auraient la capacité d'interdire l'accès au foncier n'aurait pas été viable dans un milieu dominé par l'imprévisibilité des averses et des ressources naturelles (Keen 1997: 66). Comme l'écrivait Robert Tonkinson (1991: 65) pour les Mardu, autre groupe du Désert de l'Ouest, «l'organisation locale est notable pour sa flexibilité, sa fluidité et l'absence d'insistance sur des frontières et des modes exclusifs d'appartenance à des groupes». Insister sur l'exclusivité, l'auteur souligne encore, serait autodestructif (voir aussi Poirier 1992: 759; Sackett 1975; Myers 1990). Contrairement à ce que pense Sansom (2007), avocat de l'universalité du modèle unilinéaire et partisan des sources anciennes, l'ethnographie dominante rapportée pour cette aire culturelle ne permet pas même de sousentendre un modèle patrilinéaire ni pour la situation contemporaine, ni pour une situation de précontact auxquelles les sources permettent de remonter. 
Si nous évoquons l'ethnologue Sansom (2007: 78-79), c'est parce qu'il fut l'anthropologue expert pour le Territoire du Nord, et parce qu'il défend la conclusion du juge dans le cas de Yulara. Il souligne que "personne n'a proposé un récit convaincant et réfléchi qui expliquerait pourquoi ces enquêtes anthropologiques anciennes qui produisaient et réaffirmaient de manière constante le modèle patrilinéaire, devaient maintenant être considérées comme étant "erronées" ». Pour Sansom, la formule est simple: «une règle de bon sens est de dire que les sources anciennes sont les meilleures». Nous retrouvons les convictions de Lévi-Strauss.

Nous avons déjà souligné que les sources «modernes» (depuis les années 1960) produites par l'ethnologie au sujet du Désert de l'Ouest sont, au contraire des sources anciennes, le résultat d'études de longue durée et que toutes rapportent un système foncier qui ne s'articule pas autour de groupes de descendance. Il nous faut maintenant également montrer en quoi les sources dites anciennes sont erronées. Nous allons nous attarder uniquement sur celles qui ont constitué le pilier central dans la décision du juge: les quelques notes d'Elkin et les travaux de Tindale.

Dans la transcription de l'audience de Yulara, Elkin y est décrit comme ayant effectué un «terrain extensif» ( $(130)$. Pourtant, il est bien connu que les travaux de Elkin suivaient la méthode «des sondages rapides» (Burke 2005: 212). Rappelons-nous également que c'est à la suite de ces enquêtes brèves et souvent superficielles que fut décrit le système de parenté dit Aluridja (celui du Désert de l'Ouest) qui avait causé tant de problèmes pour la théorie de la parenté. Rappelons aussi que c'est à la demande de Radcliffe-Brown qu'Elkin enquêta en 1930 auprès de nombreuses tribus et groupes de l'État de l'Australie du Sud.

Ainsi, Elkin ne constitue pas un véritable problème dans notre argumentation. Premièrement, parce que son ethnographie est pauvre; deuxièmement, parce qu'il n'affirme jamais l'existence de groupes de descendance unilinéaires. Le cas de Tindale est plus délicat. Naturaliste de formation, Norman B. Tindale fut particulièrement intéressé par l'aspect territorial des sociétés aborigènes et entreprit un travail de cartographie de longue haleine sur plusieurs décennies, traçant les frontières géographiques des tribus australiennes (1940, 1974). À plusieurs reprises il visita brièvement des groupes du Désert de l'Ouest et y enregistra quelques généalogies, photographia des individus, cartographia des sites et enregistra les totems des personnes. Combinant ces totems à la toponymie, il conclut à l'existence de clans totémiques qui seraient patrilinéaires et qui seraient l'assise de l'organisation territoriale. Selon ce système, un père possède un totem et par ce biais un territoire, et il transmet les deux à ses fils.

Peter Sutton et Petronella Vaarzon-Morel (2003), les anthropologues experts dans le cas de Yulara représentant les Aborigènes, reprirent l'ensemble des notes de Tindale et montrèrent devant la Cour que les cas pour lesquels le fils avait un totem identique au père étaient pourtant extrêmement rares. En outre, les publications de Tindale et notamment celle qui fut centrale et citée par le juge dans le 
cas de Yulara (Tindale 1988) ne s'inspirent que très peu de ses propres notes ethnographiques. Plutôt que d'analyser les données pourtant riches dont il disposait, Tindale extrapola des traits isolés afin de reconstruire une société traditionnelle conforme aux modèles anthropologiques dominants. Par exemple, Tindale formula des conclusions inexactes sur l'origine des catégories sociales dans cette région. Il parla de clans localisés sans décrire leur nature ou indiquer leurs noms, alors que, par ailleurs, il fournit une carte détaillée comprenant de nombreux toponymes indigènes. Il expliqua également que le Pitjantjatjara était en train de devenir la lingua franca de la région tout entière (nous attendons encore aujourd'hui que ceci arrive). Il avança que la langue du Désert de l'Ouest était très ancienne, alors que les linguistes la datent de moins de mille cinq cents à deux mille ans (McConvell 1990), etc. Enfin, il avança que les Pitjantjatjara étaient divisés en petits groupes patrilinéaires qui héritaient leurs totems par filiation. Cette formulation est singulièrement intrigante lorsque l'on sait que les seuls véritables totems à l'œuvre dans cette région sont les totems de conceptions et que, au moins depuis Frazer et Durkheim, nous savons aussi que l'on n'hérite pas ce genre de totem. En analysant les données rapportées par Tindale en 1933, Sutton (2006) montre que dans neuf cas sur quatre-vingt-dix-sept seulement le totem ou le lieu de naissance étaient identiques entre père ou mère et au moins l'un de leurs enfants. Dans un cas seulement les données reflétaient le modèle unilinéaire proposé par Tindale.

Il est difficile d'expliquer pourquoi Tindale insistait sur ce modèle alors que ses propres données le contredisaient. Par contre, il est certain que pour les voisins des Pitjantjatjara, les Ngaatjatjarra, pour lesquels il indiquait un modèle socioterritorial identique, Tindale n'avait aucune donnée permettant ce genre de conclusions. Nous avons analysé les carnets de terrains $(1935,1963)$ qu'il avait rapportés de ses brèves enquêtes et nous les avons comparés à nos propres généalogies qui remontent à cette période et au-delà. Si les données généalogiques récoltées par Tindale correspondent à quelques exceptions près aux nôtres, ni les premières ni les secondes ne permettent d'entrevoir un système de descendance quelconque. Les modèles de l'organisation socioterritoriale pour lesquels Radcliffe-Brown est largement responsable prennent chez Tindale le dessus sur la réalité ethnographique. Il est parfois difficile de nager à contre-courant.

\section{Quelle preuve pour quelle fin?}

Notre brève excursion historique sur l'inclusion des modèles anthropologiques dans les textes législatifs en Australie ne doit pas faire croire au rejet d'une anthropologie capable de produire du savoir objectif. Tel n'est pas notre propos, ni ici ni ailleurs. L'objet de notre discussion est que, indépendamment des outils, processus et contextes qui permettent de produire un savoir, ce dernier 
ne devient une preuve que dans un contexte particulier. Il semblerait, comme l'expliquent Gary Edmond et David Mercer (2006), que l'idée que les sciences sont susceptibles de produire des descriptions totalement objectives dans des contextes législatifs est probablement naïve.

Les théories structuro-fonctionnalistes posèrent problème car elles furent interprétées comme des lois naturelles, sous-entendant que le modèle est équivalent à la pratique et qu'une règle locale est aussi une loi universelle. Cette interprétation fut reprise par le corps juridique pour deux raisons principales, nous semble-t-il. L'approche "naturaliste» permettait d'abord de définir un système coutumier qui fut présenté comme étant durable et universel. Ensuite, elle avança la primauté de l'ancienneté des sources sur les terrains contemporains. Les sources modernes, qui courent le danger d'inclure les transformations sociales sans s'en rendre compte et dont la jurisprudence doit justement prendre acte, sont ainsi conçues a priori comme étant souillées par un activisme anthropologique malinowskien.

Howard Morphy (2006) proposait que soient distingués par et pour la Cour deux types de participations anthropologiques. D'abord ce qu'il appelle les «faits»: une compilation systématique de données qui ne sont qu'en partie le produit d'analyses. Ensuite, les «modèles et synthèses anthropologiques», ce que certains juges ont appelé des «constructions». Il s'avère malheureusement que cette distinction n'est pas souvent possible ni même explicite, car la compilation des données est presque toujours informée par des modèles et synthèses, mais aussi car les juges interprètent trop souvent les modèles comme étant des faits observés.

Ainsi, légitimer une revendication indigène ne pose pas seulement des questions de nature épistémologiques, mais constitue en premier lieu un processus intimement lié à l'historique des approches anthropologiques et à leur validation par le corps juridique. Ces processus et mécanismes de validation dépassent largement la capacité d'action de l'expert et du groupe aborigène qui doivent construire une description socioculturelle prouvant la continuité de leurs pratiques coutumières. Dans ce conflit latent entre anthropologie moderne et acteurs de la jurisprudence, une caractéristique se confirme: les modalités du travail ethnologique sont imbriquées dans les enjeux politiques, et sa capacité à répondre à ces enjeux légitimera son existence. 


\section{Ouvrages cités}

BERNDT, Ronald M. 1959. «The concept of "The Tribe" in the Western Desert of Australia», Oceania, vol. 30, n 2: 81-107.

Burke, Paul. 2005. «Law's Anthropology: from Ethnography to Expert Testimony in Three Native Title Claims», PhD, Canberra, The Australian National University.

- 2007. «The Problem when Flexibility is the System ", Anthropological Forum, vol. 17, $\mathrm{n}^{\circ} 2: 162-165$.

Douglas, Wilfrid H. 1971. «Dialect Differentiation in the Western Desert A Comment", Anthropological Forum, vol. 3, $\mathrm{n}^{\circ} 1: 79-82$.

DousseT, Laurent. 2002. "Accounting for Context and Substance: the Australian Western Desert Kinship System", Anthropological Forum, vol. 12, n ${ }^{\circ} 2$ : 193-204. - 2003. «On the Misinterpretation of the Aluridja Kinship System Type (Australian Western Desert)», Social Anthropology, vol. 11, $\mathrm{n}^{\circ} 1: 43-61$. - 2005. Assimilating Identities: Social Networks and the Diffusion of Sections. Sydney, Oceania Publications, monograph 57. — et Katie Glaskin. 2007. "Western Desert and Native Title: How Models Become Myths », Anthropological Forum, vol. 17, $\mathrm{n}^{\circ} 2: 127-148$.

Edmond, Gary et David Mercer. 2006. "Anti-social Epistemologies", Social Sudies of Science, vol. 36, n 6: 843-853.

Elkin, Adolphus P. 1931. "The Social Organisation of South Australian tribes", Oceania, vol. 2, n 1: 44-73.

- 1938-1940. «Kinship in South Australia», Oceania, vol. 8, n 4: 419-452; vol. 9, $\mathrm{n}^{\circ}$ 1: 41-78; vol. 10, n ${ }^{\circ}$ 2: 198-234; vol. 10, $n^{\circ} 3$ : 295-349; vol. 10, n 4: 369-89.

Evans-Pritchard, Edward E. 1950. «Social Anthropology, Past and Present. The 1950 Marett Lecture», Man, vol. 50: 118-124.

GLaskin, Katie. 2003. "Native Title and the "Bundle of Rights" Model. Implications for the Recognition of Aboriginal Relations to Country", Anthropological Forum, vol. $13, \mathrm{n}^{\circ} 1: 67-88$.
- 2004. «An Anthropological Perspective on Writing for the Court», Land, Rights, Laws: Issues of Native Title, vol. 2, $\mathrm{n}^{\circ}$ 29: 1-12.

- 2007. "Manifesting the Latent in Native Title Litigation", Anthropological Forum, vol. $17, \mathrm{n}^{\circ} 2$ : $165-168$.

GuMBerT, Marc. 1984. Neither Justice nor Reason. A Legal and Anthropological Analysis of Aboriginal Land Rights. St Lucia, University of Queensland Press.

HiATT, Lester R. 1962. «Local organization among the Australian Aborigines ", Oceania, vol. 32, n 4: 267-286.

- 1968. "Ownership and Use of Land among the Australian Aborignes", in Richard B. Lee et Irwen DeVore (éd.), Man the Hunter. Chicago, Aldine: 99-102.

KeEN, Ian. 1997. «The Western Desert vs the Rest: Rethinking the Contrast», in Francesca Merlan, John Morton et Alan Rumsey (éd.), Scholar and Sceptic: Australian Aboriginal Studies in Honour of $L R$ Hiatt. Canberra, Aboriginal Studies Press: 65-93.

- 1999. «The Scientific Attitude in Applied Anthropology ", in Sandy Toussaint et Jim Taylor (éd.), Applied Anthropology: Australasia. Perth, The University of Western Australia Press: 27-59.

- 2007. «Sansom's Misreading of "The Western Desert vs the Rest" ", Anthropological Forum, vol. 17, n 2 : 168-170.

LEACH, Edmund R. 1951. «The Structural Implications of Matrilateral Cross-cousin Marriage ", Journal of the Royal Anthropological Institute, vol. 81:24-53.

LeVI-STrauss, Claude. 1967 [1947].

Les structures élémentaires de la parenté. Paris, Mouton.

— 1968. «Gidjingali Marriage

Arrangements: Comments and Rejoinder», in Richard B. Lee et Irwen DeVore (éd.), Man the Hunter. Chicago, Aldine: 210-211.

MadDock, Kenneth. 1982. "Aboriginal Land Rights Traditionally and in Legislation: A Case Study", in Michael Howard (éd.), Aboriginal power 
in Australian society. St Lucia, University of Queensland Press : 55-78.

MalinowsKi, Bronislaw. 1929. «Practical Anthropology", Africa, vol. 2, n 1:22-38.

- 1938. Methods of Study of Culture Contact in Africa. Londres, Oxford University Press for the International African Institute of African Languages and Cultures (International Institute of African Languages and Cultures. Memorandum XV).

McCALl, Daniel F. 1980. "Radcliffe-Brown vs Historical Ethnology: The Consequences of an Anthropological Dispute for the Study of Africa's Past ", The International Journal of African Historical Studies, vol 13, $\mathrm{n}^{\circ}$ 1: 95-102.

McConvell, Patrick. 1990. «The linguistic Prehistory of Australia: Opportunities for Dialogue with Archaeology", Australian Archaeology, vol. 31: 3-27.

Miller, Wick R. 1971. «Dialect Differentiation in the Western Desert Language ", Anthropological Forum, vol. 3, n $1: 61-78$.

MorpHy, Howard. 2006. «The Practice of an Expert: Anthropology in Native Title», Anthropological Forum, vol. 16, n ${ }^{\circ} 2$ : 135-151.

MorTon, John. 2007. «Sansom, Sutton and Sackville: Three Expert Anthropologists?», Anthropological Forum, vol. 17, $\mathrm{n}^{\circ} 2$ : 170-173.

Myers, Fred R. 1990. «Always Ask:

Resource Use and Land Ownership among Pintupi Aborigines of the Australian Desert», in William H. Edwards (éd.), Traditional Aboriginal Society. Melbourne, Macmillan: 96-112.

PeTERson, Nicolas. 2006. "I can't follow you on this horde-clan business at all": Donald Thomson, Radcliffe-Brown and a Final Note on the Horde», Oceania, vol. $76, \mathrm{n}^{\circ} 1: 16-26$.

PoIRIER, Sylvie. 1992. «Nomadic Rituals: Networks of Ritual Exchange between Women of the Australian Western Desert ", Man, vol. 27, $\mathrm{n}^{\circ}$ 4: 757-776.

RADCLIFFE-BRown, Alfred R. 1929. «Historical and Functional Interpretations of Culture in Relation to the Practical
Application of Anthropology to the Control of Native Peoples", 4th Pan-Pacific Sciences Congress, vol. 3 : 537-538.

- 1956. «On Australian Local

Organization ", American Anthropologist, vol. 58, n² 2: 363-367.

ReINING, Conrad C. 1962. «A Lost Period of Applied Anthropology", American Anthropologist, vol. 64, $\mathrm{n}^{\circ}$ 3: 593-600.

RuMseY, Alan. 1996. «Aspects of Native Title and Social Identity in the Kimberleys and Beyond", Australian Aboriginal Studies, vol. 1996, n 1: 2-10.

SACKeTT, Lee. 1975. «Exogamy or Endogamy: Kinship and Marriage at Wiluna, Western Australia», Anthropological Forum, vol. 4, n ${ }^{\circ}$ 1: 44-55. - 2007. "A Potential Pathway», Anthropological Forum, vol. 17, n 2 : 173-175.

SANSOM, Basil. 2007. «Yulara and Future Expert Reports in Native Title Cases ", Anthropological Forum, vol. 17, $\mathrm{n}^{\circ} 2$ : 71-92.

SuTTON, Peter. 2006. «Norman Tindale and Native Title. His Appearance in the Yulara Case», The 2006 Norman B. Tindale Memorial Lecture. Adelaide, South Australian Museum.

http://wwwling.arts.kuleuven.be/fll/eldp/sutt on/2006nbt.pdf

- 2007. «Norms, Statistics and the Jango

Case at Yulara ", Anthropological Forum, vol. 17, n² 2: 175-184.

— et Petronella VAarzon-Morel. 2003.

Yulara Antbropology Report. Alice Springs, Central Land Council.

Tindale, Norman B. 1935. «Journal and Sociological Cards of the Anthropological Expedition to Warburton Range, Western Australia, July-September 1935", Adelaide, South Australian Museum. - 1940. «Results of the Harvard-Adelaide Universities Anthropological Expedition, 1938-1939. Distribution of Australian Aboriginal Tribes: A field survey", Transactions of the Royal Society of South Australia, vol. 64, no $1: 140-231$.

- 1963. "Journal and Genealogies of Visit to the Rawlinson Range Area in the Great Western Desert, 24 October-25 November 1963 ", Adelaide, South Australian Museum. 
- 1974. Aboriginal Tribes of Australia. Berkeley, University of California Press. - 1988 [1972]. «The Pitjandjara», in Marco G. Bicchieri (éd.), Hunters and Gatherers today. A Socioeconomic Study of Eleven such Cultures in the Twentieth Century. New York; Londres, Waveland Press: 217-268.

TonkInsON, Robert. 1991 [1978]. The Mardu Aborigines: Living the Dream in Australia's Desert. New York, Holt, Rinehart \& Winston, (1re éd., The Mardudjara Aborigines: Living the Dream in Australia's Desert).

WeINER, James F. et Katie GLASKIN. 2006. « Introduction: the (re-)Invention of Indigenous Laws and Customs", The Asia Pacific Journal of Anthropology, vol. $7, n^{\circ} 1$, numéro special, J. F. Weiner et K. Glaskin (éd.), «Custom: Indigenous Tradition and Law in the Tweny-First Century»: 1-13.

- 2007. «Customary Land Tenure and Registration in Papua New Guinea and Australia: Anthropological Perspectives", in J. F. Weiner et K. Glaskin (éd.), Customary Land Tenure and Registration in Papua New Guinea and Australia: Anthropological Perspectives. Canberra, The Australian National University Press: 1-14. 